\title{
La liste pour écrire, et un peu plus. Modèles de référence en formation d'enseignants du premier degré
}

Micheline Cellier et Patrick Demougin

\section{QpenEdition}

\section{Journals}

Édition électronique

URL : http://journals.openedition.org/trema/1663

DOI : $10.4000 /$ trema. 1663

ISSN : 2107-0997

Éditeur

Faculté d'Éducation de l'université de Montpellier

Édition imprimée

Date de publication : 1 juillet 2000

Pagination : 43-54

ISSN : 1167-315X

Référence électronique

Micheline Cellier et Patrick Demougin, « La liste pour écrire, et un peu plus. Modèles de référence en formation d'enseignants du premier degré », Tréma [En ligne], 17 | 2000, mis en ligne le 01 juillet 2000, consulté le 19 avril 2019. URL : http://journals.openedition.org/trema/1663 ; DOI : 10.4000/ trema.1663

Ce document a été généré automatiquement le 19 avril 2019

Trema 


\title{
La liste pour écrire, et un peu plus. Modèles de référence en formation d'enseignants du premier degré
}

\author{
Micheline Cellier et Patrick Demougin
}

\begin{abstract}
« Il y a dans l'idée que rien au monde n'est assez unique pour ne pas pouvoir entrer dans une liste quelque chose d'exaltant et de terrifiant à la fois. »

PÉREC G. : Penser/classer.
\end{abstract}

1 Cet article essaie d'articuler un bilan et des perspectives. Les années 1980 étaient celles de la lecture, les années 1990 celles de l'écriture. Comment se termine aujourd'hui cette pièce dont nous connaissons les deux premiers actes, mais dont il faut écrire le troisième? La question se pose tout particulièrement en formation d'enseignants du premier degré, car, si les pratiques de classe en matière de lecture et d'écriture évoluent à leur propre rythme et de manière assez différenciée, on est en droit d'attendre des pratiques de formation une homogénéité et des orientations plus marquées.

\section{Outils à la disposition des formateurs}

2 Les approches sur la question de l'écrit sont, depuis les Instructions Officielles de 1985, associées au lire - écrire. Il n'est plus question de distinguer ces deux entités historiquement disjointes que sont la lecture et l'écriture. Depuis cette date fondatrice, où texte libre et rédaction disparaissaient des états de service didactiques, les travaux sur la production d'écrits, pris dans la logique du lire - écrire, se sont référés à deux modèles : l'un centré sur le produit, l'autre sur l'activité du sujet dans le processus de production.

Dans le premier domaine, les travaux de Jean-Michel Adam sur la typologie des textes deviennent, dans les années 1990, une référence très largement diffusée. Ils ont l'avantage, en formation d'enseignants, d'impliquer une réflexion sur la lecture et de faire référence à des catégories linguistiques. Ces deux caractéristiques sont parfaitement sécurisantes pour le formateur et le formé : il suffit de prolonger, en formation initiale, 
ou de réactiver, en formation continue, le discours linguistique dont s'inspire l'approche typologique, discours parfaitement adapté à des situations transmissives et aux savoirs savants qu'il véhicule. Le sentiment d'être dans la théorie est, somme toute, très confortable, et les conséquences dans la pratique d'enseignement sont loin d'être nulles : tris de textes, établissement de critères, grilles d'auto-évaluation sont autant d'outils largement redevables aux travaux typologiques, ceux de Jean-Michel Adam et de bien d'autres. Par ailleurs, la grammaire de textes et les réflexions sur l'énonciation trouvant dans ces perspectives matière à s'investir, on est souvent tenté de voir dans ces typologies une possible rédemption de l'enseignement de la grammaire. La tentation du tout linguistique n'est pas loin, et c'est une des limites que le colloque de Toulouse, consacré aux théories du texte, en mars 1998, a mise en évidence : la dérive formaliste à propos des textes s'est développée avec les approches typologiques.

Relevant d'une approche du sujet, la psychologie cognitive s'est engouffrée dans la brèche ouverte par le modèle de Hayes et Flower (1980) et a tenté de modéliser le processus d'écriture : sur le plan didactique, les réflexions sur l'aide procédurale (C. GarciaDebanc, M. Mas ; 1986) ont tiré le meilleur parti de cette modélisation. Dans une pratique de formation d'enseignants, ces travaux permettent de montrer les modalités précises d'une articulation entre la lecture et l'écriture. En outre, ils soulignent l'aspect dynamique et structuré du processus d'écriture, rendant ainsi possible le passage à une mise en forme de l'activité didactique. Cette perspective se révèle complémentaire de la première : elle l'est sur le plan du modèle théorique (il n'y a pas incompatibilité mais bien plutôt complémentarité entre le modèle typologique centré sur l'objet et le modèle de l'aide procédurale centré sur la pratique du sujet); elle l'est aussi dans la pratique de formation puisqu'elle vient compléter une théorie du texte par une théorie de sa production, sollicitant ainsi très directement ses implications didactiques. On touche, avec cette approche, ce qu'on pense être la réalité de l'expérience scolaire, son déroulement dans le temps et la mise au point d'outils concrets, parmi lesquels grilles et schémas sont les plus utilisés. Avec ces deux modèles, la didactique se pense comme un scénario qui enchaîne séquences et objets. Les deux premiers actes de notre pièce se complètent assez bien et s'écrivent, pour une part, simultanément.

Le troisième acte a bien failli s'écrire en dehors de notre pièce : les années 2000 sont celles de l'oral et l'accent est mis dans les travaux de recherche sur ce nouvel objet, oublié depuis les années 1970. Pour autant, les recherches en matière de lecture et d'écriture ne sont pas suspendues, et il faut bien faire évoluer les modèles proposés en formation. Dans cette logique, des recherches moins globalisantes ont mis en évidence les aspects cognitifs et langagiers de l'activité du scripteur, à propos de types de textes particuliers ${ }^{1}$ ou dans l'approche de certaines phases du processus rédactionnel : les problèmes de mise en texte à partir de difficultés très circonscrites ${ }^{2}$ et la grande question de la révision des textes à partir de perspectives psycholinguistiques ou de travaux en génétique du texte ${ }^{3}$ ont ainsi enrichi les deux modèles exploités. Produit et processus sont alors dépendants de facteurs cognitivo-langagiers. Mais il faut reconnaitre que cette mise en relation, très productive pour analyser les situations d'enseignement, ne se constitue pas en modèle didactique, faute d'être immédiatement transposable dans des outils. Elle reste souvent cantonnée à des points de vue critiques ou strictement scientifiques. De ce fait, elle reste relativement peu exploitée en formation d'enseignants. 


\section{Un dispositif éclaté, des pratiques contestables}

6 Le dispositif décrit pour le primaire est pris en défaut de plusieurs manières qui menacent sa cohérence. Outre les divers modèles scientifiques auxquels il se réfère, il n'est, en effet, animé par aucune vision claire des enjeux attachés à l'écriture et de ses spécificités par rapport à la lecture. Le décalage très fort entre les divers modèles didactiques auxquels se réfère l'enseignement $d u$ français ${ }^{4}$, selon les niveaux de scolarisation, est révélateur de ces flottements : la maternelle, outre son intérêt ancien pour le geste graphique et les recherches en matière de dictée au maître, s'intéresse surtout à l'écriture sous l'angle de la psychologie génétique (Ferreiro ; 1988, 1990). Dans cette perspective, écrire c'est, avant tout, faire évoluer une représentation de l'écrit. L'attention est portée sur des unités limitées (lettres, mots, suites de mots). L'exploitation de ce modèle en formation débouche sur l'observation des productions d'enfants et donne des informations importantes sur l'apprentissage de la lecture. Si l'on écrit, c'est essentiellement pour arriver à lire.

7 Pour l'école élémentaire, le modèle didactique que transmettent les centres de formation est inspiré par les approches linguistiques et psycho-cognitives telles que nous venons de les décrire. L'accent est mis sur les tâches liées à la production du texte, qu'elles renvoient à de la gestion globale (planification, type de texte, cohérence, ancrage énonciatif) ou à de la gestion locale (maîtrise orthographique, syntaxe, enchaînements). Dans cette perspective, tous les types d'écrit sont abordés, fonctionnels ou littéraires, et la lecture est associée à l'activité de production, dans une sorte de dialectique bienheureuse ${ }^{5}$.

Productions d'élève. Listes écrites dans la classe, pour se souvenir, pour organiser, pour se construire.

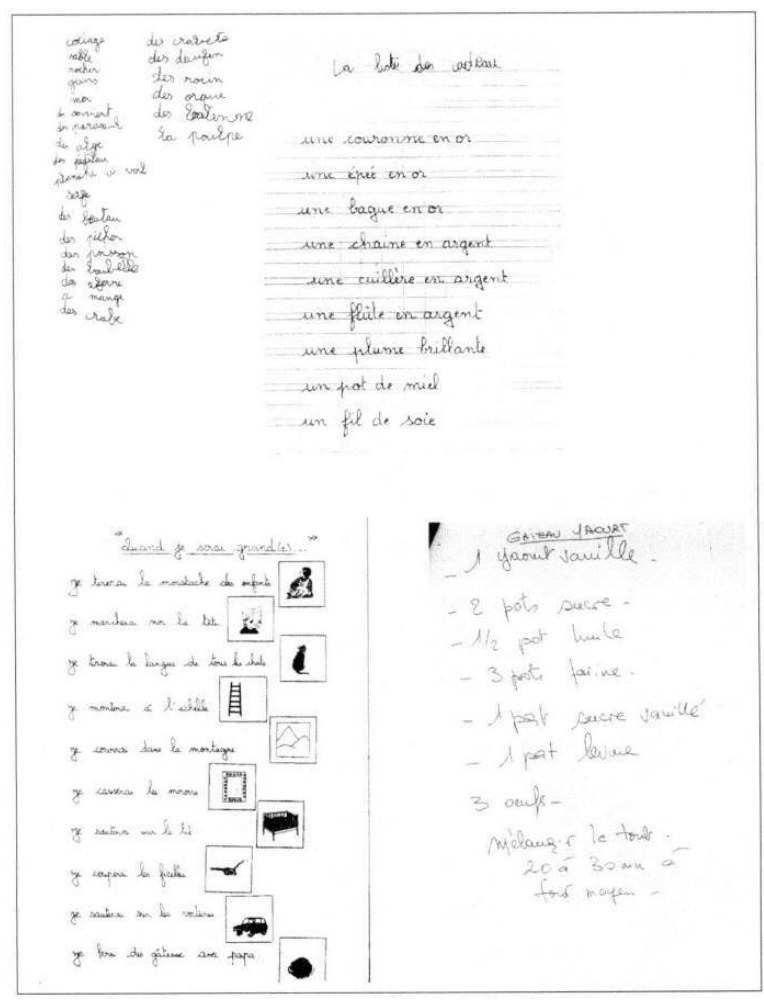


Dans l'enseignement secondaire, le modèle opératoire reste, avec bien des réserves et des atermoiements, celui de la pratique de la littérature : écrire est une activité qui réfère, à travers des exercices scolaires comme le commentaire composé, la dissertation, ou les travaux d'imagination, à l'objet littéraire. La lecture n'y est pas véritablement associée : elle existe sous la forme orale de l'explication de textes et intervient peu dans les exercices écrits. Les nouvelles perspectives ouvertes avec l'introduction d'écrits argumentatifs ne change pas radicalement les choses: au collège et au lycée, on écrit pour écrire.

9 Ballottée entre ces divers modèles, la didactique de l'écriture a quelques raisons de flotter et le discours des formateurs est, par la force des choses, à géométrie variable, suivant les publics formés. Ce serait bien légitime si ces variations étaient liées à des besoins différents formulés par les formés. Ce l'est moins si ces variations sont le fait de modèles concurrents liés à l'histoire de la formation et s'ils témoignent d'une réelle hésitation de l'institution quant aux finalités attachées à l'enseignement de l'écriture.

10 Le formalisme des approches typologiques et le cadre sécurisant de l'aide procédurale deviennent, en l'absence d'une perception claire des enjeux attachés à l'écriture, les seules bouées auxquelles se rattachent les jeunes formés. Les conséquences dans leur pratique d'enseignement sont parfois contestables. La plus fréquemment observée est l'applicationnisme, qui consiste à exploiter des fragments de théories et de dispositifs, dans des situations d'enseignement, sans véritable projet cohérent. On voit alors fleurir grilles de réécriture, schémas narratifs et fiches d'aide, un peu au hasard des expériences. On est dans le règne du «faire »: l'outil cache souvent la finalité réelle de l'activité. Une autre conséquence négative tient à la confusion entre théorie et métalangage : l'emprunt d'outils conceptuels à diverses disciplines de référence peut se résumer dans bien des cas à l'adoption approximative d'un métalangage, en formation d'abord, puis, à peine adapté, dans la pratique d'enseignement. On est alors dans le règne du «dire »: déclarer que « dans un récit il y a une situation initiale et une situation finale » tient lieu de réflexion sur le récit et vaut pour guide dans la pratique d'écriture. Les réflexions de G.D. Brassart (1995) à propos de la didactique du français en général sont valides pour ce qui concerne la didactique de l'écriture : la logique de la transposition didactique ne peut aboutir à l'acquisition d'un savoir procédural ou, pour reprendre la question autrement, "le métalangage ne vaut pas compétence procédurale "

11 L'éclatement des modèles théoriques et didactiques (qui en soi est probablement préférable à un tout linguistique ou un tout cognitif) ne permet pas au formé de se projeter dans la durée et la continuité de l'apprentissage de l'écriture; les pratiques applicationnistes privilégient le métalangage au détriment de véritables appropriations théoriques. Ces constats critiques ne sont imputables ni aux formés ni aux outils de formation, mais bien à la conception même de ce qu'est la didactique de l'écriture.

\section{Déplacer la question}

12 S'il n'est pas question de se pourvoir d'un métalangage supplémentaire, ni d'une théorie de rechange, il est intéressant, en revanche, de poser la question hors du champ didactique, du côté des recherches sur l'écriture qui trouvent peu de réinvestissement en didactique de l'écriture. Deux démarches semblent particulièrement riches et pourtant 
toujours réservées, dans la pratique de formation, à des informations sur l'écriture. Elles n'accèdent pas au statut d'outil de formation.

La première démarche est celle inspirée par les travaux à dominante sociologique ou psycho-sociologique. Les travaux de B. Lahire $(1993,1995)$ d'un côté, ceux de J-Y. Rochex de l'autre (Bautier, Charlot, Rochex; 1993) soulignent les liens étroits qui unissent rapport à l'école, rapport au savoir et rapport à l'écrit. Penser la didactique de l'écriture c'est aussi penser, dans la logique de ces travaux, aux formes d'écrits et aux savoirs transmis par l'écrit. C'est prendre en compte la réalité scolaire et ses finalités profondes : la formation du sujet par un processus culturel.

seconde démarche est celle ouverte par l'anthropologie avec les travaux de Jack Goody (1979). En montrant que l'écriture développe historiquement de nouvelles capacités cognitives et qu'elle se manifeste par l'apparition de la pensée abstraite et rationnelle, cette réflexion en souligne les fonctions essentielles: l'écriture organise la pensée et construit la mémoire. Ces deux fonctions, mnémonique et organisatrice de la pensée, permettent de former l'hypothèse que d'autres entrées dans l'écriture que celles habituellement dévolues aux pratiques de l'aide procédurale sont possibles.

Cela revient à postuler les points suivants :

- écrire permet au sujet de se construire, socialement, culturellement mais aussi sur les plans intellectuel et affectif ;

- écrire n'est possible que quand le sujet a accédé à une théorie personnelle de l'écrit, une représentation structurée de l'acte, qui lui permet d'en évaluer l'efficacité sociale et culturelle, mais aussi pour lui-même, dans son devenir ;

- apprendre à écrire débouche en dernière analyse sur une structuration de la pensée. Les savoirs procéduraux et les connaissances académiques sur l'écrit ne sont que des éléments facilitateurs exploitables quand la finalité principale de l'acte est perçue.

Les conséquences premières de ces postulats sont les suivantes: la dimension communicative, les approches formalistes, les entrées culturelles et sociales restent valides. Mais il faut aussi penser à des formes textuelles transversales présentes dans tous les types d'écrits, historiquement et théoriquement fondatrices, techniquement accessibles à l'élève. Déplacer la question, c'est se demander si, à côté des techniques héritées de modèles linguistiques et psycho-cognitifs, il n'y a pas place pour un modèle anthropologique: cela reviendrait à identifier dans l'univers scolaire des formes textuelles susceptibles de pratiques réflexives et qui fourniraient à l'élève une théorie implicite de son rapport à l'écriture et au savoir, qui, en d'autres termes, lui fourniraient une modélisation possible mettant en relation son activité de scripteur et son développement intellectuel, sans qu'il soit nécessaire de passer par un savoir savant. En ce sens, seraient atténués l'éclatement théorique et les risques applicationnistes évoqués plus haut. Il ne s'agirait plus tant de transmettre un type de texte ou de développer la compétence du scripteur que d'installer, avant tout, une pratique langagière et sa nécessaire mise en forme théorique. C'est à une didactique qui ne transpose pas qu'il convient de s'adresser. Une didactique qui n'offre pas pour argent comptant le confort du métalangage et des outils prêts à l'emploi, qui ne vise pas des compétences. Une didactique de l'écriture qui vise, en deçà des grands objectifs affichés, à lier une pratique langagière et une activité conceptuelle. 


\section{En quête d'un écrit prototypique : l'hypothèse de la liste}

17 Dans cette perspective, Jack Goodyl ${ }^{7}$ fournit une hypothèse de travail : les premières formes textuelles sont des listes. D'une certaine manière, la liste est l'écrit prototypique qui concentre les fonctions principales de l'écriture. C'est historiquement et anthropologiquement acceptable, si l'on souscrit aux thèses de Jack Goody. Mais peut-on fonder, en didactique de l'écriture, une démarche englobante telle que celle que nous envisageons sur cette forme textuelle ? C'est cette question qu'il convient d'examiner.

La première remarque qui s'impose est que la liste appartient à l'univers scolaire. Des listes de mots, aux conjugaisons, aux règles de vie en classe, en passant par les listes de matériel scolaire à acheter, le registre des absences, certains poèmes énumératifs, la préparation d'un débat... la liste est un outil extrêmement répandu dans la vie de la classe, mais rarement interrogé dans ses fonctions didactiques. On s'en sert comme outil scolaire, pour accomplir diverses tâches.

19 Si on l'examine en fonction du sujet et de ses apprentissages, la liste accompagne le développement cognitif de l'élève. Les enfants du cycle II ont recours à des listes pour tester leur tout nouveau savoir-faire en matière d'écriture. Plusieurs sondages auprès de classes de GS et de CP montrent que les brouillons d'élèves ou les écritures spontanées se construisent souvent sous la forme de listes, d'abord horizontales, puis verticales. Plus tard, on observe au cycle III des usages très techniques de la liste pour rassembler des idées, présenter des personnages ou des actions avant une tâche rédactionnelle. On retrouve aussi dans les prises de notes, lors de travaux documentaires en $B C D$, un usage fréquent de la liste. Il est clair qu'elle permet de vérifier des savoir-faire et de mettre en forme des savoirs au fur et à mesure que se développe la pratique scolaire. Il faut noter aussi qu'elle est une forme d'écrit intime et familial : les listes des courses et le journal intime recourent à la liste ${ }^{8}$.

Par ailleurs, la liste recoupe tous les types de textes et d'écrits. C'est une autre caractéristique essentielle de son fonctionnement : elle assure une sorte de transversalité entre toutes les catégories de l'écrit. Elle est bien sûr très présente dans tous les écrits sociaux (annuaire, journal, registre, catalogue...). Elle fonctionne à l'intérieur de types de textes clairement identifiés dans leur diversité, dans la liste des ingrédients du texte injonctif qu'est la recette, dans le texte explicatif avec les listes de synonymes, les listes dans les encyclopédies, les listes descriptives sur l'étiquette d'un produit. Elle apparaît aussi dans le texte littéraire avec la poésie énumérative ou dans le paratexte avec les listes des personnages au théâtre. Elle constitue, implicitement, la structure de certains récits. Elle est à la fois infratexte, paratexte et texte à part entière.

Enfin, la question de sa définition n'est pas première, ce qui évite de s'enfermer très vite dans une approche typologique savante. Il est seulement utile de la reconnaître. Lors de tests d'identification avec des publics adultes on note une convergence très forte des réponses: on accepte ou refuse comme liste, souvent sans les lire, les divers textes proposés. Il apparaît très vite, aux yeux des publics testés, que la liste a des traits linguistiques et textuels relativement simples. Nous pouvons, après avoir utilisé des formes ambiguës (listes rédigées horizontalement, éclatées dans des dispositions complexes sur des documents publicitaires, tableaux) proposer les critères suivants de 
reconnaissance sur lesquels se sont accordées les personnes testées: elle se manifeste dans une disposition formelle verticale, respectant de nombreux blancs graphiques à droite, selon un ordre des mots indépendant d'enchainements syntaxiques. En affinant l'observation, des caractéristiques secondaires ont été mises en place: absence de détermination dans le GN, ou détermination répétée (numéraux, possessifs); priorité à des structures nominales (nominalisations, substantifs, avec présence faible d'expansions à droite) ou des structures phrastiques correspondant chacune à une ligne ; des principes d'organisation chronologiques, alphabétiques, numériques, référentiels et logiques (ordre des priorités dans les appels d'urgence sur le calendrier des pompiers, par exemple).

Ces caractéristiques formelles facilitent l'accès à l'écriture dans la mesure où les problèmes de mise en texte (Hayes et Flower) ou, sur un autre plan, les difficultés liées aux opérations de linéarisation (Schneuwly) sont fortement minorés. Chez les enfants, elle fait l'objet d'une reconnaissance diffuse et parfois d'un rejet au début du primaire (c'est une écriture "de bébé»). On connaît donc la liste d'abord parce qu'on s'en sert, comme la marche à pied, et elle est à peu près aussi simple à définir. Sinon qu'à mettre un pied devant l'autre, on préférera y mettre un mot au dessous de l'autre.

\section{Un écrit qui n'empêche pas de penser}

La liste permet de concentrer le travail du scripteur sur une phase particulière de la production d'écrit. La planification devient l'objet essentiel de l'activité et se trouve élargie à tout ce qui concerne la mise en ordre du monde. Dans une pratique rédactionnelle se joue l'organisation d'une pensée, par la sélection et la hiérarchisation d'informations. 
Listes usuelles et listes poétiques. La liste organise l'expérience humaine dans ses aspects les plus variés.

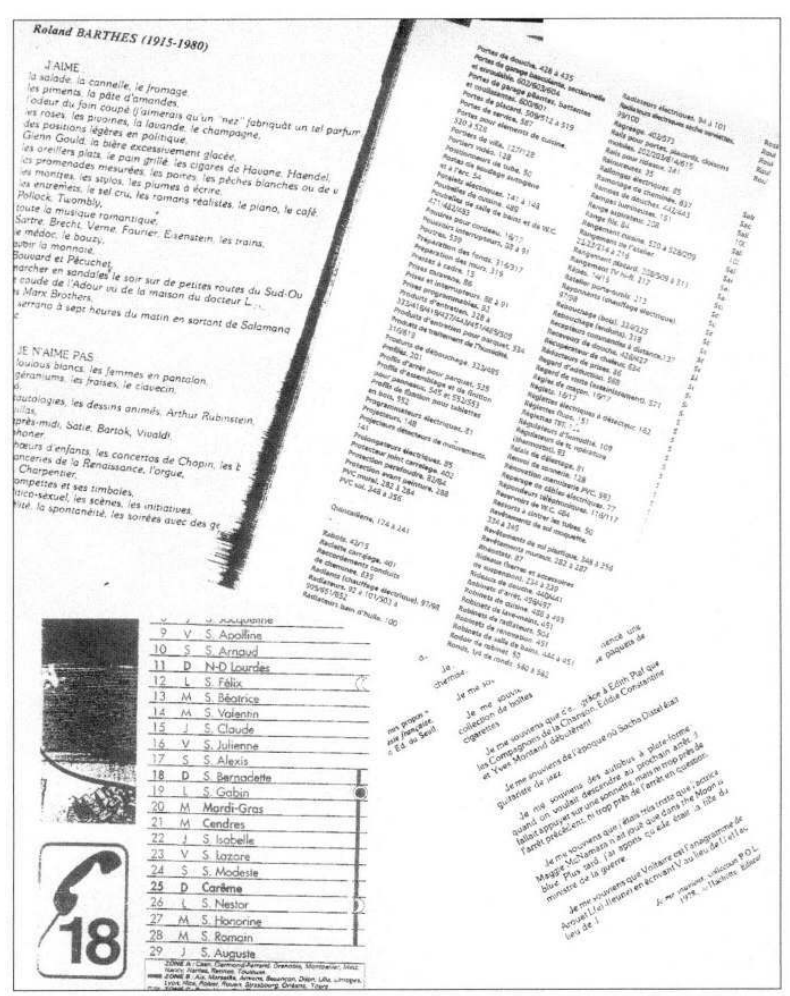

Dans le cadre de la mise en place du texte descriptif, la liste organise l'aspectualisation; dans la narration, elle anticipe sur certains aspects structuraux du récit (chronologie des événements, liste de personnages, scénario); dans le texte argumentatif, elle organise l'argumentaire. Mais elle peut aussi, outre ses vertus apéritives, suffire à ses propres fins, du poème à la Prévert aux tables de la loi. Dans chaque situation d'écrit, elle associe au plus près activité mentale et activité rédactionnelle. Elle rejoue, sur un mode individuel et non plus historique, l'avènement d'une raison graphique, conformément aux hypothèses de J. Goody.

Quelques corollaires se dessinent alors : le mot devient, dans la liste, la matérialisation très forte de ce lien entre activité mentale et activité rédactionnelle. Hors des questions syntaxiques et des contraintes liées à la cohésion textuelle, il manifeste très directement l'évidence du sens. Ensuite, il est évident que la liste appelle, beaucoup plus que les textes développés, à une attitude interprétative : lire une liste oblige à reconstruire des liens, à faire des hypothèses nécessaires. Enfin, la liste permet de mettre en relation des fonctionnements langagiers très divers: entre l'oral, qu'elle peut noter même si Jack Goody écarte cette hypothèse, et les écrits complexes, elle appelle constamment à la reformulation. Ces caractéristiques en font typiquement un écrit pour apprendre.

Mais il faut se garder de cette appellation qui peut simplement ajouter aux modèles didactiques dont nous disposons un volet méthodologique : on travaillerait, à côté des habituelles démarches en production d'écrits, un écrit particulier utile aux tâches scolaires, au même titre que certaines formes de l'oral. Cette perspective est réductrice et ne modifie pas significativement les données didactiques. En revanche, porter à la liste, dans tous ses aspects, littéraires et fonctionnels, scolaires et intimes, transitoires ou non, une attention particulière revient à formuler, avec la question de l'écriture, l'exigence d' 
une didactique spécifique aux fonctionnements langagiers. Elle ne saurait se concevoir dans les seules formes, communes aux autres disciplines, de la transposition didactique et des démarches par objectifs. Elle suppose un travail du sujet sur lui-même. $E t$, en conséquence, une formation d'enseignants qui prépare plus à l'analyse des situations langagières et à l'individualisation des démarches, qu'à l'application d'outils, aussi performants soient-ils. Elle suppose aussi de ne pas toujours faire écrire selon les formes normées de la communication écrite, pour laisser se construire, avec la liste, des formes plus proches de l'activité mentale du sujet: petits papiers, griffonnages, brouillons, écrits intimes... toutes formes qui peuvent apparaitre spontanément, mais qui demandent aussi un regard professionnel de la part des enseignants.

Ce changement de point de vue n'est envisageable que si, à côté des démarches usuelles en production d'écrits et des modèles de référence bien connus, on fait émerger un nouveau modèle didactique centré sur la dimension anthropologique. Dans quelle mesure l'écrit me permet d'organiser mon temps, mon espace, ma pensée, comment, aussi, il m'autorise à prendre position, à faire valoir un point de vue ? Ce sont là les questions qu'il faut faire apparaître, et qui ne le peuvent guère dans le cadre des démarches actuelles. Elles postulent que l'écrit, avant d'être impliqué dans des formes culturellement construites, est une activité humaine qui participe à la construction de la personne. La liste est, parmi d'autres, la forme exemplaire de ces écrits non normés qui participent très directement à la formation humaine. En ce sens, écrire c'est, pour paraphraser Montaigne, plus que « former l'homme », le « réciter », et la liste peut être la cheville ouvrière de cette ambition, elle qui n'est jamais que l'anagramme du «stile " cher au même auteur.

\section{BIBLIOGRAPHIE}

BAUTIER E., CHARLOT B. et ROCHEX J.Y. : Ecole et savoir dans les banlieues et ailleurs. Paris, A. Colin, 1993.

BRASSARD G.D. : « Approche(s) cognitiviste(s) ? », in - CHISS J.L., DAVID J. et REUTER Y, (dir.) :

Didactique du français, Etat d'une discipline. Paris, Nathan, 1995.

BRONCKART J.P. et alii : Le fonctionnement des discours. Neufchâtel (Suisse), Delachaux et Niestlé, 1985.

DENHIERE G. : Il était une fois... .Lille, PUL, 1984.

FABRE C. : Les brouillons d'écoliers. Grenoble, Ceditel - l'atelier du texte, 1990.

FABRE C. : «Ce qui vaut pour un manuscrit d'écrivain vaut-il pour un brouillon d'écolier? », in Le français Aujourd'hui, N 108, 1994.

FAYOL M. :Le récit et sa construction. Neufchâtel (Suisse), Delachaux et Niestlé, 1985.

FAYOL M. : « Une approche psycholinguistique de la production », in Langue française, $\mathrm{N}^{\circ} 81$, 1989. 
FAYOL M. : « Apprendre à produire des textes. Tentative d'intégration des apports de la psychologie cognitive et de la psycholinguistique textuelle : la production de récits ", in - BARRÉDE MINIASC. (dir.) : Vers une didactique de l'écriture. pour une approche pluridisciplinaire. Paris Bruxelles, Éd. conjointe : INRP - De Bœck, 1996.

FERREIRO E., GOMEZ PALACIO M. : Lire écrire à l'école : comment s'y apprennent-ils ?, (trad.). Lyon, CRDP, 1988.

FERREIRO E. : Apprendre le lire - écrire. Lyon, Voies Livres, 1990.

GOODY J. : La raison graphique : la domestication de la pensée sauvage. Paris, Editions de minuit, 1979.

HAYES J.R., FLOWER L. : “Identifying the organization of writing processes", in - GREGG W., STEIBERG E. : Cognitive processes in writing.Hillsdale (état du New Jersey, USA), Lawrence Erlbaum \& Associates Publishers, 1980.

LAHIRE B. : Culture écrite et inégalités scolaires. Lyon, PUL, 1993.

LAHIRE B. : Tableaux de familles. Paris, EHESS - Gallimard - le Seuil, 1995.

ORIOL-BOYER C. : L'écriture du texte : théorie, pratique, didactique. Université Paris VIII, thèse de doctorat d'Etat, 1989.

ROUBAUD J. : « trois ruminations », in Bibliothèque oulipienne, $\mathrm{N}^{\circ} 81,1996$.

VIGOTSKY L. : Pensée et langage, (trad.). Paris, Editions sociales, 1985.

\section{NOTES}

1. Sur le récit: DENHIERE $(1984)$, FAYOL $(1985,1996)$; puis des travaux, a partir de la fin des années quatre-vingts, sur les textes descriptifs, argumentatifs, explicatifs (Pratiques, $\mathrm{N}^{\circ} 55,51$, 58 ; Repères $\left.\mathrm{N}^{\circ} 72,77\right)$. Toutes ces approches relativisent les aspects formalistes et proposent de nouveaux modèles centrés sur des aspects langagiers.

2. Par exemple : usage des connecteurs (FAYOL, 1989), ancrage dans le contexte du point de vue de l'énonciation (BRONCKART, 1985).

3. Travaux de Claudine FABRE sur les brouillons d'élèves $(1990,1994)$ et de Claudette ORIOLBOYER (1989).

4. Voir sur ce point l'état de la question propose dans Didactiques du français sous la direction de JL. CHISS, J.DAVID et Y. REUTER (Nathan 1995)

5. Lire pour écrire et écrire pour lire deviennent les deux faces de cette dialectique. Voir les propositions de Bernard DEVANNE (Lire et écrire des apprentissages culturels, tomes I et II, A. Colin, 1992) ou Jacques BERNARDIN (comment les enfants entrent dans la culture écrite, Retz, 1997)

6. Didactiques du français, 1995, Nathan, p. 126.

7. La raison graphique, Editions de Minuit, 1986.

8. Marie Claude Penloup, «la liste non-utilitaire, vers la prise en compte didactique de cette pratique d'écriture extra-scolaire », in Repères $\mathrm{N}^{\circ} 15,1997$. 
AUTEURS

MICHELINE CELLIER

IUFM de Montpellier

\section{PATRICK DEMOUGIN}

IUFM de Montpellier 